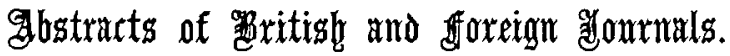

\section{Model of the upper part of the Spinal Cord and the} Oblongata. ${ }^{1}$ By Dr. Ludwig Edirater, Frankfort-on-the-Maine.

THE knowledge of the inner structure of the central nervous system is generally acquired by examining and combining transverse sections. As the delineations of these impress themselves especially on the minds of students, I have found it advisable, in my new model, to represent the fibres as issuing from the reproduction of a transverse cut.

A revolving pillar rises from a heavy pedestal; to the top of this is attached a horizontal bar- $75 \mathrm{~cm}$. long-representing the central canal. To one end of the bar is fastened a perforated metal plate on which the rhomboidal area of the medulla is painted, whereas the other end of the bar terminates in a similar reproduction of the cervical part of the spinal cord. The various nuclei and fibres are distinguished from one another by different colours. The proportions as well as the colouring have been so selected that minute details can be seen at a considerable distance.

The most important fibres and cells have been reproduced by means of wires and wooden models. Between the cross sections a number of variously painted wires are extended which represent connections of identical areas or continuation of the entering root fibres.

The cells of the spinal ganglia and of the motor and sensory nuclei are shown in their various forms. Larger aggregations of cells, of which it seems difficult to give a proper imitation in a model, as for example the forms of the nuclei of the posterior columns and the ascending gelatinous column of the trigeminus and glossopharyngeus are simply given in carved wood. In order

\footnotetext{
'Author's translation from his paper in Anatomischer Anzeiges', 1893, No. 5.
} 
not to make the matter too complicated the nuclei of the hypoglossal and vagus are given in the same form.

As the model is designed to give a first insight into the subject, I have endeavoured to make it as simple as possible, and several tracts consisting only of a few fibres have been omitted. The name of each part is signified by a printed label. (In the wood cut, which illustrates this description, numbers are substituted.)

As few colours as possible have intentionally been used, and each tract retains its colour throughout. All motor root tracts are painted light red, the primary sensory tracts light blue. The secondary motor tracts are dark red, the central sensory tracts dark blue.

All the wires representing tracts which extend either caudad or frontad beyond the sections project at either end.

The model was completed in the spring of 1892 , and shows matters as they were treated in the third edition of my "Vorlesungen ueber den Bau der nervösen Centralorgane," Leipzig, C. W. Vogel.

In studying the model it is the best plan to begin with an anterior root (light red wire, No. 32 in the cut). Motor roots enter into the transverse sections as well as into the intermediate, part of the model, but only the cross section gives a complete view of the different ways they take. Every root fibre terminates in a multipolar ganglion cell. Round this cell twines a branch of fine red wires, the extremity of the secondary motor tract (red wires) which springs as a collateral either from the lateral (No. 26) or anterior (27) pyramidal tract. It is easy, in this manner, to follow these pyramidal fibres as far as the base of the medulla where the lateral tract decussates and both together form the ventral pyramidal area (No. 19) of the oblongata.

Of the sensory nerve (light blue, No. 30) a short peripheric portion is given, the greater part of whose fibres terminate in cells of the spinal ganglion (31). From these cells the roots enter the model and divide in the well known manner into different branches. Each part, however, is here represented by a single fibre only. The division of entering roots into ascending and descending branches is shown. One part of the posterior roots enters the posterior tracts $(20,21)$ in which it ascends and can be traced as far as the posterior nucleus funiculatus of the medulla. Another reaches the grey matter through the posterior grey column where its fibres either spread themselves around the cells or proceed to the Clarke's column. One fibre can be traced up to a cell of the anterior grey column. The direct tracts leading 
to the limiting layer of the grey substance as well as the fibres extending to the crossed posterior tract have been omitted; likewise the posterior commissure, because our knowledge of the structure of the latter is too imperfect.

From the fusiform cells (dark blue) of the posterior column springs the secondary sensory tract (dark blue). It decussates through the anterior commissure into the antero-lateral tract, and may be followed up to the fillet (11). The (yellow) fibres of the lateral cerebellar tract spring from the cells of Clarke's column. 25 shows the position of the fasciculus antero-lateralis at the central margin of the above-mentioned lateral cerebellar tract. Lastly, in the spinal cord a single commissural cell is shown which sends its dividing fibre to the antero-lateral tract of the opposite side.

The secondary tracts which spring from the nucleus of the funiculus cuneatus and funiculus gracilis in the medulla are the following: On the mesial side the internal arciform fibres (15) crossing to the fillet, and laterally the external arciform fibres anterior and posterior $(13,14)$ to the restiform body $(8,9)$. So, by following the blue fibres it is easy to demonstrate the rather complicated course of the sensory tract as it is thought to be at the present time. Thus it strikes the eye that in the medulla all fibres are situated on the side opposite to that which they have entered in the root. Laterally from the oblongata cross section the accessory nerve (33), which may be followed to its knee, juts out. Of other cranial nerves, the ascending part of the trigeminus (6) and of the glossopharyngeus (7) is shown: their fibres, dividing into fine brushes, end in the gelatinous columns, which in our model are represented in carved wood. Besides, the hypoglossal nerve (18) and part of the vagus roots $(16,17)$ are represented; from the nuclei of the latter the secondary (central) fibres are seen crossing over to the fillet. Of the connection between medulla and cerebellum only the spinal part of the restiform body could be shown, because the fibres descending from the cerebellum to the olivary body (10) only appear in more central parts of the medulla.

N.B.-As only part of the fibres have been embodied in our model, a few hanging wires had to be supported by metal props. These props, as all other parts of the model which serve merely technical purposes, have been painted in black, in order to be distinguished from the essential wires.

Both cross-sections bear handles which are to be used for turning the model, in order not to dirty the bright colours by touching. 
ABSTRACTS OF BRITISH AND FOREIGN JOURNALS. 461

The model is manufactured in the laboratory of Mr. R. Jung at Heidelberg. It is durable, and solidly as well as neatly made; with the excepiion of the nuclei and gelatinous sub. stance, it is constructed entirely in metal. Care has been taken, in designing it, that new wires may easily be inserted, as well as other changes made.

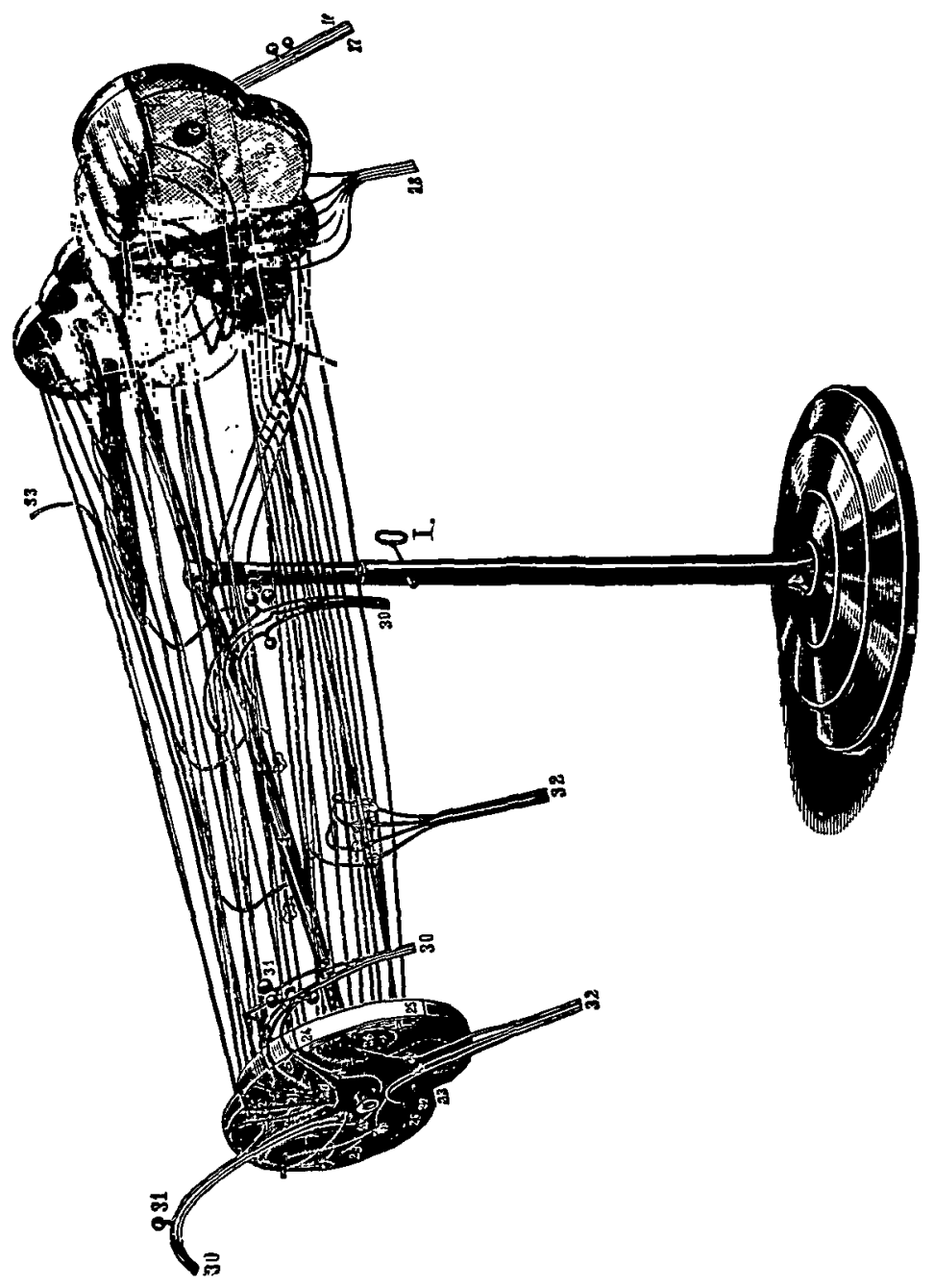

